University of Nebraska - Lincoln

DigitalCommons@University of Nebraska - Lincoln

Gordon Gallup Publications

Research Papers in Physics and Astronomy

2009

Semiclassical Propagation Method for Tunneling Ionization

Ilya I. Fabrikant

University of Nebraska-Lincoln, ifabrikant@unl.edu

Gordon A. Gallup

University of Nebraska-Lincoln, ggallup1@unl.edu

Follow this and additional works at: https://digitalcommons.unl.edu/physicsgallup

Part of the Physics Commons

Fabrikant, llya I. and Gallup, Gordon A., "Semiclassical Propagation Method for Tunneling Ionization" (2009). Gordon Gallup Publications. 50.

https://digitalcommons.unl.edu/physicsgallup/50

This Article is brought to you for free and open access by the Research Papers in Physics and Astronomy at DigitalCommons@University of Nebraska - Lincoln. It has been accepted for inclusion in Gordon Gallup Publications by an authorized administrator of DigitalCommons@University of Nebraska - Lincoln. 


\title{
Semiclassical propagation method for tunneling ionization
}

\author{
Ilya I. Fabrikant and Gordon A. Gallup \\ Department of Physics and Astronomy, University of Nebraska, Lincoln, Nebraska 68588-0111, USA
}

(Received 3 November 2008; published 8 January 2009)

\begin{abstract}
We apply the semiclassical propagation technique to tunneling ionization in atomic and molecular systems. Semiclassical wave functions and the tunneling flux are calculated from the solution of the classical equations of motion in the complex time plane. We illustrate this method by rederiving the known result for the decay rate of a negative ion in a weak electric field. We then obtain numerical results for atomic hydrogen, $\mathrm{H}_{2}{ }^{+}, \mathrm{H}_{2}$, and $\mathrm{Ar}$, and compare them with the results of the asymptotic [Ammosov-Delone-Krainov (ADK)] theory. The asymptotic theory gives surprisingly good results for the atomic and molecular ionization rates. In particular, our calculations for the simplest case of molecular suppression, ionization of $\mathrm{H}_{2}$ versus Ar, confirms the ADK analysis of Tong et al. [Phys. Rev. A 66, 013409 (2002)], explaining that the suppression is mainly due to the different symmetries of the ionized orbitals, $s$ in $\mathrm{H}_{2}$ and $3 p_{z}$ in $\mathrm{Ar}$.
\end{abstract}

DOI: 10.1103/PhysRevA.79.013406

PACS number(s): $32.80 . R m, 33.80 . R v, 32.60 .+\mathrm{i}$

\section{INTRODUCTION}

Tunneling ionization of atoms and molecules is usually described by the asymptotic theory of Smirnov and Chibisov [1] and Perelomov et al. [2]. Ammosov et al. [3] showed that this theory can be applied to ionization in an alternating field, if the field frequency is small enough [Ammosov-DeloneKrainov (ADK) theory]. The asymptotic theory assumes that there is a region of space where the external electric field can be neglected, but at the same time the atomic wave function already reaches its asymptotic expression (in a.u.),

$$
\psi_{l m}(\mathbf{r})=C_{l} r^{Z / \kappa-1} e^{-\kappa r} Y_{l m}(\hat{\mathbf{r}}),
$$

where $l m$ is the orbital angular momentum and its projection, $Z$ is the charge of the atomic residue, $\kappa=\sqrt{-2 \mu E} / \hbar, \mu$ is the electron mass, and $E$ is the electron energy. $(|E|$ is the ionization potential.) Therefore this theory is limited by the condition $F r_{0} \ll|E|$, where $F$ is the electric force on the electron, and $r_{0}$ the characteristic size of the atomic or molecular system. In addition, the theory uses an approximation for the tunneling integral for the motion in the combined Coulomb field and electric field,

$$
S_{0}=\int_{z_{1}}^{z_{2}} d z\left[2 \mu\left(-E-F z-\frac{\alpha}{z}\right)\right]^{1 / 2},
$$

where $\alpha=Z e^{2}$ is the Coulomb constant, $z_{1}, z_{2}$ are classical turning points. In the asymptotic theory the expression for the tunneling integral can be obtained by the semiclassical perturbation method [4] with the result

$$
S_{0}=\frac{\mu^{1 / 2}(-2 E)^{3 / 2}}{3 F}-\frac{\alpha \mu}{\hbar \kappa} \ln \left(2 \frac{\hbar^{2} \kappa^{3}}{\mu F}\right),
$$

which is valid only for $F \ll \hbar^{2} \kappa^{3} / \mu$. Note that Eq. (3) is exact for $\alpha=0$, i.e., in the case of the negative-ion decay.

In Fig. 1 we present comparison of three expressions for the tunneling integral $S_{0}$ for the hydrogen atom. Inclusion of the Coulomb term by the perturbation theory noticeably improves the $\alpha=0$ result, but disagreement with the exact result becomes substantial at $F>0.03$. Noting that the ionization rate depends on the tunneling integral exponentially, we con- clude that the asymptotic theory should fail very badly for the electric fields above 0.03 a.u. Nevertheless, it has been used for quite a long time for analysis of the data on strongfield ionization of atoms and molecules [5-13]. Moreover, these applications are quite often extended into the electric field range where the whole concept of tunnelinng becomes inapplicable because of barrier suppression, and the theory for above-barrier ionization [14] should be used. The barriersuppressed ionization (BSI) occurs above $F_{B S I}=E^{2} / 4 \alpha$, which equals 0.0625 a.u. in the example of Fig. 1 .

A review of the quoted papers leads to a paradoxical conclusion. From Fig. 1 one would expect that the asymptotic (ADK) theory strongly underestimates the actual ionization rate whereas in reality it typically overestimates it. Comparisons with ab initio calculations [7,15] demonstrate that the asymptotic theory agrees with $a b$ initio calculations within $20 \%$ at $F<F_{B S I}$, an astonishing conclusion in view of Fig. 1.

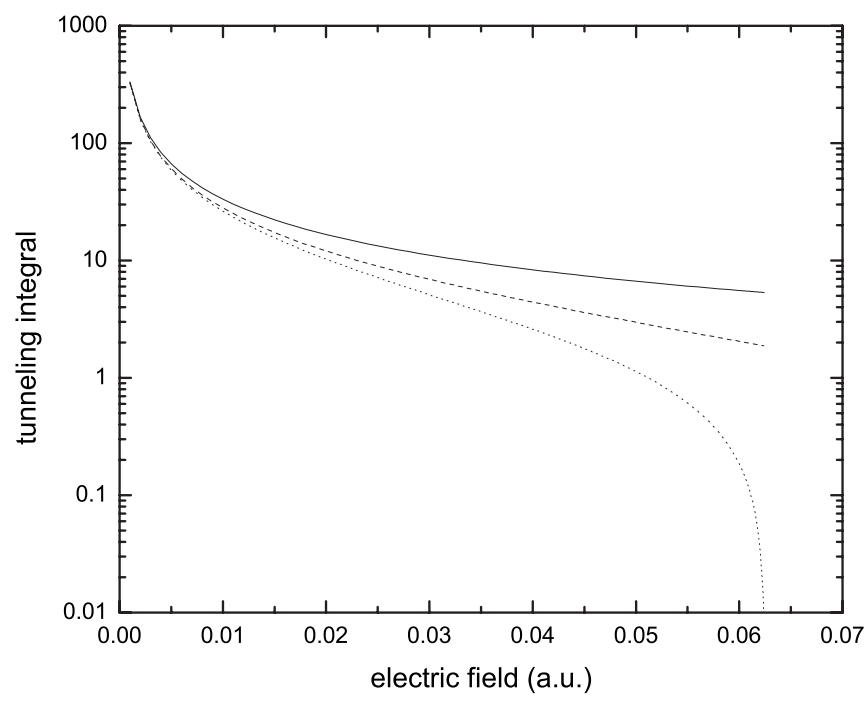

FIG. 1. Tunneling integrals for the ionization of the hydrogen atom. Solid line-tunneling integral without the account of the Coulomb interaction; dashed line-inclusion of the Coulomb interaction by the first-order perturbation theory, Eq. (3); dotted linethe exact tunneling integral. 
Moreover, different researchers mean by "ADK" different versions of the asymptotic theory which makes comparisons even more confusing. The original asymptotic theory $[1,2]$ contains a coefficient $C_{l}$ in Eq. (1) which should be calculated from the $a b$ initio atomic (or molecular) wave function. However, Ilkov et al. [16] suggested an extension of the hydrogenic formula for $C_{l}$ which has been used since then in other calculations $[5,15]$. This extension simply replaces quantum numbers $n$ and $l$ in the hydrogenic formula by noninteger values obtained from experimental energies. Obviously, this procedure introduces further uncertainties in what is called ADK theory.

Since most applications of the tunneling theory deal with atomic and molecular ionization in an ac field, it is necessary to know how low a field frequency is required to make the static-field limit valid. This is usually estimated from the value of the Keldysh parameter [17]

$$
\gamma=\omega \frac{\sqrt{-2 \mu E}}{F_{0}}
$$

where $\omega$ is the frequency of the ac field, and $F_{0}$ is the electric force amplitude. The tunneling ionization regime corresponds to a small $\gamma$. The Keldysh parameter can be interpreted as a ratio of the tunneling time to the period of the laser field. However, the tunneling time in Eq. (4) corresponds to the motion in a short-range (strictly speaking, zero-range) potential and does not take into account the influence of the Coulomb field. Moreover, in complex molecules this time can be substantially affected by the electronic structure [18]. DeWitt and Levis [18] calculated a modified Keldysh parameter (which they call the adiabaticity parameter), derived from the actual barrier width in $\mathrm{Xe}, \mathrm{N}_{2}$, and $\mathrm{C}_{6} \mathrm{H}_{6}$, and the corresponding tunneling probabilities. As one can expect from Fig. 1, the tunneling probability has increased by several orders magnitude (five orders for xenon and nitrogen and seven orders for benzene). The molecular effect is particularly noticeable in benzene. The ratios of modified tunneling probabilities calculated by DeWitt and Levis [19] for benzene, naphthalene, and anthracene agree with their measurements.

This issue might be relevant to the ionization suppression phenomenon observed in several molecules. From the ADK theory one might expect that the ionization rates for two targets with the same ionization potential should be roughly equal. This was confirmed by early experiments on molecular ionization $[5,20]$. More recent experiments brought substantial corrections. Ionization of the hydrogen molecule was found to be substantially suppressed compared to ionization of the "companion" atom $\mathrm{Ar}$ [21]. Ionization of $\mathrm{O}_{2}$ is suppressed relative to its "companion" atom Xe [6,22]. In contrast, ionization of $\mathrm{N}_{2}$ is not suppressed $[6,22]$ compared to the "companion" Ar atom.

There were several attempts to explain these observations. A dissociative recombination mechanism suggested in [22] turned out to be untenable [6]. Another approach [8,21] explains the molecular suppression by orientational and related effective charge effects. This explanation requires an empirical effective charge adjustment. Saenz [23] investigated vi- brational effects, but they turned out to be small compared to the observed suppression. An alternative theory [24] explains suppression in $\mathrm{O}_{2}$ by interference effects. These authors argue that due to different symmetries of the valence orbitals, the interference in $\mathrm{O}_{2}$ is destructive, whereas in $\mathrm{N}_{2}$ it is constructive. This theory predicts suppression in $\mathrm{F}_{2}$. However, the subsequent experiment for $\mathrm{F}_{2}$ [9] did not confirm this prediction.

A comprehensive analysis of the ability of the ADK theory to explain observed suppressions was done by Tong et al. [10]. They separated the ratio of molecular rate to the "companion" atomic rate into three factors, $A_{1}, A_{2}$, and $A_{3}$. The first factor is due to electronic structure reflected in the asymptotic coefficient $C_{l}$ in Eq. (1) and its generalization for molecular case. In the latter case it also contains the result of orientational average. The second factor $A_{2}$ is the ratio of the preexponential factors in transition rates which depends only on $F, Z, m$, and the binding energy $E$. The factor $A_{3}$ is the ratio of the exponential factors of the asymptotic theory, $\exp \left(-2 S_{0}\right)$, where $S_{0}$ is given by Eq. (3) with $\alpha=0$. Tong et al. explained the $\mathrm{D}_{2}$-Ar suppression mostly by the factor $A_{1}$, but also partly by the factor $A_{3}$ which reduces to 0.42 if the vertical ionization energy, rather than adiabatic ionization energy is used. The most important reduction, $A_{1}=0.31$ appears due to different symmetries of the ionized orbitals, basically $s$ for $\mathrm{D}_{2}$ and $p_{z}$ for Ar. For $\mathrm{N}_{2}$, however, the valence orbital $\sigma_{g}$ deviates substantially from isotropic due to a substantial $d$ contribution that results in no $\mathrm{N}_{2}$-Ar suppression. The $\mathrm{O}_{2} / \mathrm{Xe}$ suppression is explained by a small value of $A_{2}$ which is due to the $m=1$ projection of the valence $\pi_{g}$ orbital on molecular axis. This explanation works, however, only if the molecule aligned along the external field. For a random orientation no such suppression should be expected. Finally, for the $\mathrm{F}_{2}$ : Ar ratio again strong suppression is obtained due to the small value of $A_{2}$, whereas the experiment [9] does not indicate any suppression.

It is clear that the problem is far from being solved. It is even not clear yet if there is one general mechanism for suppression that is able to explain all observations, or whether the problem should be solved on a case by case basis.

The purpose of the present paper is twofold. First, in view of an increase in tunneling rate of several orders of magnitude due to Coulomb and molecular effects, we want to analyze how reliable the ADK theory is. Second, by developing a more accurate theory of tunneling ionization, we want to investigate in detail the physics of the molecular suppression effect. For this purpose we need a method that incorporates the correct tunneling factor and takes into account the molecular structure including the orientational effects in the molecular charge distribution. At the same time we want the method to be simple enough to extend it to polyatomic molecules with moderate modifications of existing molecular structure codes. We have found that the semiclassical propagation technique [25-27] extended into the complex time plane is quite convenient for this purpose. The complex time method for tunneling ionization problems was proposed by Perelomov et al. [2] and then extensively used by the same group in various atomic physics problems [4,28] (see also a recent review [29]). It can be also used for a description of 
collision processes [30]. For application to complex atomic and molecular systems one has to go beyond approximations necessary in analytical approach used in Refs. [2,4,28].

We will start with the semiclassical propagation method and will illustrate it by a simple problem which can be solved analytically, detachment of negative ions in a weak electric field. Then we will verify the validity of our method by applying it to tunneling ionization of atomic hydrogen. Finally, we will demonstrate it by the calculation of ionization of $\mathrm{H}_{2}{ }^{+}, \mathrm{H}_{2}$, and by comparing the latter ionization rate with that of Ar.

\section{SEMICLASSICAL PROPAGATION METHOD}

We propose to use the semiclassical propagation technique $[26,27]$ which connects the wave function $\psi_{0}(\mathbf{q})$ given on a closed surface $S_{0}$ with the wave function at some arbitrary (not necessarily closed) surface by the equation (for a time-independent Hamiltonian, $\hbar=1$ ),

$$
\psi(\mathbf{r})=\sum_{k} \psi_{0}\left(\mathbf{q}^{(k)}\right) A_{k}\left(\mathbf{r}, \mathbf{q}^{(k)}\right) e^{i\left[S_{k}(\mathbf{r}) / \hbar-\mu_{k} \pi / 2\right]},
$$

where the index $k$ enumerates classical trajectories emerging from the surface $S_{0}$ perpendicular to this surface. The initial coordinates on the surface are given by $\mathbf{q}^{(k)}$, and the final point is r. $\mu_{k}$ is the Maslov index that counts the number of intersections with caustics [31], and $S_{k}$ is the reduced action for trajectory $k$, that is $($ action $+E t)$, where $E$ is the total energy of the system.

The semiclassical amplitude

$$
A(\mathbf{r}, \mathbf{q})=\sqrt{\left|J_{t \rightarrow 0}(\mathbf{r}, \mathbf{q}) / J_{t}(\mathbf{r}, \mathbf{q})\right|}
$$

with

$$
J_{t}(\mathbf{r}, \mathbf{q})=\partial(\mathbf{r}(t)) / \partial(t, \mathbf{q}) .
$$

This Jacobian probes the dependence of a trajectory end point $\mathbf{r}(t)$ on the time of flight $t$ and on the coordinates $\mathbf{q}$ of the starting point on the initial surface.

In the atomic and molecular ionization problem it is natural to choose the surface $S_{0}$ as a sphere surrounding the atom or the molecule. For strong fields $\psi(\mathbf{q})$ can be calculated with the account of external field. It can be easily done using a quantum chemistry computational program, placing additional point charges far from the center of the molecule, so that it produces appropriate uniform electric field within the surface $S_{0}$. Then we use Eq. (5) and the action, either in a pure electric field (for negative-ion detachment) or in superposition of the Coulomb and uniform electric field (for ionization). This allows us to find the wave function and electron flux at large distances. The flux gives the ionization rate.

For the tunneling ionization the classical motion through the barrier is forbidden. Therefore classical trajectory equations should be analytically continued in the complex time plane [30].

\section{A. Negative-ion decay}

We will illustrate these ideas now by rederiving the formula of Smirnov and Chibisov [1] for detachment of a nega- tive ion. Although the imaginary time method was used for this problem in Ref. [2], our approach is somewhat different and more convenient for the extension to complex atomic and molecular systems.

Assume first that the classical motion is allowed. If we assume that the classical trajectories emerge from a small sphere of radius $r_{0}$, then the equations of motion are (with the force $F$ along positive $z$ axis)

$$
\begin{gathered}
v_{z}=v_{0} \cos \theta+\frac{F}{\mu} t, \\
x=\left(r_{0}+v_{0} t\right) \sin \theta \cos \phi, \\
y=\left(r_{0}+v_{0} t\right) \sin \theta \sin \phi, \\
z=\left(r_{0}+v_{0} t\right) \cos \theta+F t^{2} / 2 \mu,
\end{gathered}
$$

where $\mu$ is the electron mass, $\theta$ is the ejection angle, and $v_{0}$ is the initial electron velocity. Excluding time from here, we obtain relation between two possible escape angles $\theta_{1,2}$ and the trajectory final point $(\rho, z) \rho^{2}=x^{2}+y^{2}[32]$,

$$
\cot \theta_{1,2}=\frac{l}{\rho}\left(-1 \pm \sqrt{1+2 z / l-\rho^{2} / l^{2}}\right),
$$

where $l=\mu v_{0}^{2} / F$, and for the reduced action [32]

$$
\begin{aligned}
S= & \frac{2^{1 / 2}}{3} \mu v_{0} l\left(1+\frac{z}{l} \mp \sqrt{1+\frac{2 z}{l}-\frac{\rho^{2}}{l^{2}}}\right)^{1 / 2} \\
& \times\left(2+\frac{2 z}{l} \pm \sqrt{1+\frac{2 z}{l}-\frac{\rho^{2}}{l^{2}}}\right) .
\end{aligned}
$$

The first sign in Eqs. (12) and (13) describes the "downstream" trajectory corresponding to the shortest path between the source and the observation point $(\rho, z)$. The second sign corresponds to the "upstream" trajectory. For the tunneling problem only the first trajectory is important. Furthermore, in the process of tunneling ionization the flux is mostly concentrated along the $z$-axis, therefore we will need the expansion of Eq. (13) at small $\rho$,

$$
S=\frac{1}{3} \mu v_{0} l\left[\left(1+\frac{2 z}{l}\right)^{3 / 2}-1\right]+\frac{\rho^{2}}{2 l^{2}} \frac{\mu v_{0} l}{\sqrt{1+2 z / l}-1} .
$$

Now we will assume $v_{0}^{2}<0$ and perform analytical continuation into the classically forbidden region for the "downstream" trajectory. Since in the subbarrier region kinetic energy is negative, both $v_{0}$ and $t$ in Eq. (9) should be pure imaginary,

$$
\begin{gathered}
v_{0}=i v, \quad t=i \tau, \\
v_{z}=i v \cos \theta+\frac{F}{\mu} i \tau, \\
z=-v \tau \cos \theta-\frac{F \tau^{2}}{2 \mu},
\end{gathered}
$$




$$
\rho=-v \tau \sin \theta
$$

At the classical turning point for $\rho=0, z_{t}=\mu v^{2} / 2 F$ we should have $v_{z}=0$, therefore $v$ and $\tau$ should be of opposite signs. Calculating now the action for $z=z_{t}, \rho=0$, we have

$$
S\left(z_{t}, 0\right)=\frac{i \mu^{2} v^{3}}{3 F} .
$$

The semiclassical propagator should decay in the positive $z$ direction, therefore $v>0$ and $\tau<0$. For $z>z_{t}$, $t$ becomes complex.

Introducing now the parameter $\lambda=-l=\mu v^{2} / F$, we can rewrite Eq. (14) in the form

$$
S=-\frac{1}{3} i \mu v \lambda\left[\left(1-\frac{2 z}{\lambda}\right)^{3 / 2}-1\right]-\frac{\rho^{2}}{2 \lambda^{2}} \frac{i \mu v \lambda}{\sqrt{1-2 z / \lambda}-1} .
$$

We now analytically continue this expression into the classically allowed region $z>z_{t}=\lambda / 2$. In doing this we have to choose the correct branch for the function $(1-2 z / \lambda)^{1 / 2}$. Let us consider the relation between $t$ and $z$ for $\rho=0$,

$$
t=\frac{i \mu v}{F}\left(\sqrt{1-\frac{2 z}{\lambda}}-1\right) .
$$

At $z>\lambda / 2, t$ should have a positive real part growing proportionally to $\sqrt{z}$ at large $z$, therefore we choose the $-i$ branch of the square root,

$$
t=\frac{i \mu v}{F}\left(-i \sqrt{\frac{2 z}{\lambda}-1}-1\right) .
$$

Accordingly, Eq. (20) turns into

$$
S=\frac{1}{3} \mu v \lambda\left(\frac{2 z}{\lambda}-1\right)^{3 / 2}+\frac{i}{3} \mu v \lambda+\frac{i \mu v \rho^{2}}{4 z}\left[1-i\left(\frac{2 z}{\lambda}-1\right)^{1 / 2}\right] \text {. }
$$

With this action we have to calculate the current density at $z \rightarrow \infty$,

$$
J=\frac{1}{\mu} \operatorname{Im}\left(\psi^{*} \frac{d \psi}{d z}\right)=\frac{1}{\mu}\left|\psi_{0}(\mathbf{q}) A(\mathbf{r}, \mathbf{q})\right|^{2} e^{-2 \operatorname{Im} S} \frac{d \operatorname{Re} S}{d z} .
$$

The rate is obtained by integrating $J$ over a plane perpendicular to the $z$ axis,

$$
\Gamma=2 \pi \int J(\rho) \rho d \rho .
$$

The semiclassical amplitude $A$ in Eq. (5) can be calculated from the general Eq. (6), but it also can be taken from Ref. [32]. By doing analytical continuation, we obtain

$$
A=\frac{r_{0}}{r}\left[\frac{1-\frac{z}{\lambda}+\sqrt{1-\frac{2 z}{\lambda}-\frac{\rho^{2}}{\lambda^{2}}}}{2 \sqrt{1-\frac{2 z}{\lambda}-\frac{\rho^{2}}{\lambda^{2}}}}\right]^{1 / 2} \text {. }
$$

$$
A=\frac{r_{0}}{r}\left(-\frac{z}{8 \lambda}\right)^{1 / 4}
$$

and the amplitude becomes independent of $\rho$. Since the initial wave function $\psi_{0}$ depends on $\rho$ via the ejection angle $\theta$, Eq. (12), we now have to investigate the angular dependence of $\psi_{0}$. The asymptotic theory of Smirnov and Chibisov [1] uses the following expression for $\psi_{0}$ [cf. Eq. (1)],

$$
\psi_{0}(\mathbf{r})=C e^{-\kappa r} r^{-1} N P_{l}^{m}(\cos \theta),
$$

where $P_{l}^{m}$ is the Legendre function, $\kappa=\sqrt{-2 \mu E}=\mu v, C$ is an asymptotic coefficient, and

$$
N=\left[\frac{2 l+1}{4 \pi} \frac{(l-m) !}{(l+m) !}\right]^{1 / 2} .
$$

Since the current density is decreasing very fast with $\rho$, we now take the limit of small $\theta$

$$
P_{l}^{m}(\cos \theta)=\frac{(-1)^{m}(l+m) !}{2^{m} m !(l-m) !}(\sin \theta)^{m},
$$

and

$$
\psi_{0}(\mathbf{r})=C \frac{e^{-\kappa r}}{r} \frac{(-1)^{m}}{2^{m} m !}\left[\frac{2 l+1}{4 \pi} \frac{(l+m) !}{(l-m) !}\right]^{1 / 2}(\sin \theta)^{m}
$$

At small $\theta$ from Eq. (12)

$$
\tan \theta=i \frac{\rho}{(2 z \lambda)^{1 / 2}},
$$

therefore $\psi_{0}$ is proportional to $\rho^{m}$, and integration over $\rho$ is reduced to the integral

$$
\int_{0}^{\infty} e^{-2 \operatorname{Im} S} \rho^{2 m+1} d \rho=\exp \left(-\frac{2}{3} \mu v \lambda\right) \frac{m !}{2}\left(\frac{2 z}{\mu v}\right)^{m+1} .
$$

The factor $r_{0}$ in Eq. (27) is canceled by $1 / r_{0}$ in the wave function, Eq. (28). Substituting Eq. (33) in Eq. (25), we obtain

$$
\Gamma=\frac{C^{2}(2 l+1)}{2(2 v)^{m}} \frac{(l+m) !}{m !(l-m) !}\left(\frac{F}{2 \kappa^{2}}\right)^{m+1} \exp \left(-\frac{2}{3} \kappa \lambda\right) .
$$

This result coincides with that of Smirnov and Chibisov if we correct it by replacing $m$ ! in their Eq. (11) by $1 /\left(2^{m} m !\right)$, as was indicated by Perelomov et al. [2].

\section{B. Tunneling ionization of neutral atoms}

The same approach can be extended to neutral systems if we take into account the long-range Coulomb interaction between the escaping electron and the residual ion. However, the analytical result is valid only for weak fields. To obtain the analytical formula presented in Refs. [1,2] we have to assume first that the atomic wave function in the asymptotic region, Eq. (28), is not affected by the electric field. Second, calculation of the action integral in the presence of the Coulomb field is reduced to the elliptic integral [33], and in order to obtain a simpler analytical expression for the tunneling integral, used in the analytical theory $[1,4]$, weak-field approximation is necessary. 
To correct these drawbacks of the asymptotic theory, we will assume that the atomic wave function outside the atom on the sphere of radius $r_{0}$ is given by a polarized orbital $\psi^{(0)}+\psi^{(1)}$, where $\psi^{(1)}$, in the first-order perturbation theory, is a solution of the equation

$$
\left(-\frac{1}{2 \mu} \nabla^{2}+V(r)-E^{(0)}\right) \psi^{(1)}=F r \cos \theta \psi_{0},
$$

where $V(r)$ is the potential due to the ionic residue, and $E^{(0)}$ and $\psi_{0}$ are the energy eigenvalue and eigenfunction in the absence of the field. The solution for $\psi^{(1)}$ is well known for the hydrogen atom (in a.u.) [34],

$$
\psi^{(1)}(\mathbf{r})=\frac{\cos \theta}{\sqrt{4 \pi}}\left(2 r+r^{2}\right) e^{-r} .
$$

The resulting wave function is propagated from the spherical surface by numerical integration of the classical equations of motion in the presence of the Coulomb and the static fields. From the previous section it is clear that time and action acquire imaginary parts when integration is carried out in the classically forbidden region. Consider, for example, the motion in the Coulomb field along the $z$ axis, $V(z)=-\alpha / z$. There are two turning points $z_{1,2}$ given by the solution of the equation

$$
E+F z+\frac{\alpha}{z}=0 .
$$

If we start integration in the region $z<z_{1}$, then the imaginary part of $t$, acquired at large $t$ is

$$
t_{0}=-\int_{z_{1}}^{z_{2}} \frac{d z}{v(z)}, \quad v(z)=\left[\frac{2}{\mu}\left(-E-F z-\frac{\alpha}{z}\right)\right]^{1 / 2},
$$

and a similar expression holds for $\operatorname{Im} S$, Eq. (2). Since integration is performed numerically, no approximation for the tunneling integral is involved in this procedure. A possible integration path is going along the real $t$ axis, then turning parallel to the imaginary axis until $\operatorname{Im} t=i t_{0}$ is reached, and then continuing parallel to the real axis. It is shown as path 1 in Fig. 2

If the initial integration point $z_{0}$ is located in the classically forbidden region, we start on the imaginary axis with the initial imaginary $t$ corresponding to $z_{0}$. The corresponding integration path is shown as path 2 in Fig. 2. In fact this approach is always used in practice, since in the region $z$ $<z_{1}$ non-Coulomb molecular effects are almost always important, except some obvious cases, such as ionization of atomic hydrogen.

The final result does not depend on the shape of the integration path, therefore it can be arbitrary. In practice it is convenient to parametrize the integration path in the form

$$
\operatorname{Re} t=\xi, \quad \operatorname{Im} t=t_{0}^{\prime} e^{-\xi / \beta}+t_{0}, \quad t_{0}^{\prime}=\int_{z_{0}}^{z_{2}} \frac{d z}{v(z)},
$$

where $\beta$ is a parameter making a smooth transition from the starting point to the asymptotic region (integration path 3 in Fig. 2). The integration is subject to the condition that the observable position coordinates $\rho$ and $z$ are reached at the

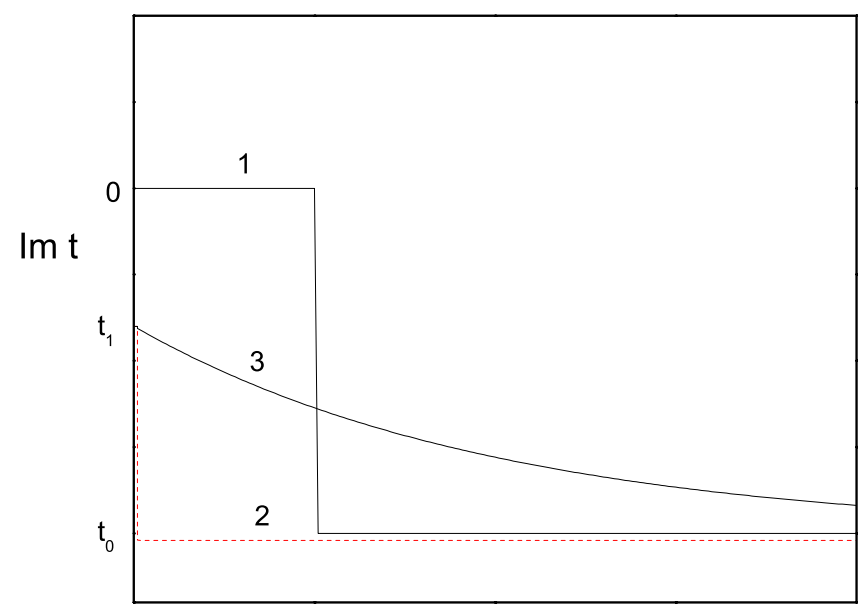

$\operatorname{Re} t$

FIG. 2. (Color online) Integration paths in the complex $t$ plane as discussed in text. Note that $t_{1}=t_{0}^{\prime}+t_{0}$ with $t_{0}$ and $t_{0}^{\prime}$ given by Eqs. (38) and (39), respectively.

end of the path. Therefore we have to solve the equations for $\theta$ and $t$,

$$
\rho(\theta, t)=\rho, \quad z(\theta, t)=z,
$$

where $\rho$ and $z$ are real. In contrast, the roots of these equations, initial angle $\theta$ and time $t$, are not observable and are complex. As follows, for example, from Eq. (32), in the negative-ion detachment problem at small $|\theta|, \theta$ is pure imaginary. This might cause some difficulties in calculation of the initial wave function, since it is defined in the real coordinate space. However, if the electric field is not too weak, only a very small range of the incident angles $\theta$ contributes to the electron flux, and we can perform the current density integration either assuming $\theta=0$ (for $m=0$ ) or performing analytical continuation in $\theta$ similar to Eq. (32) (for $m \neq 0$ ).

When the energy of the initial state approaches the top of the potential barrier, the whole approach starts to fail, of course. Comparison with quantum-mechanical results for the ground-state hydrogen atom shows that the semiclassical ionization rate becomes substantially lower than the exact result. If the energy is not very close to the top of the barrier, the semiclassical result can be improved by taking into account multiple reflections at the classical turning points. For example, if the trajectory, after passing the classically forbidden region, is reflected at the right turning point $z_{2}$, and then is reflected again at the left turning point $z_{1}$, the semiclassical wave function is multiplied by the factor $\exp \left(-2 S_{0}\right)$ where $S_{0}$ is given by Eq. (2) Summing over all possible reflections, we obtain the following correction factor $f$ in the wave function

$$
f=\frac{1}{1-\exp \left(-2 S_{0}\right)},
$$

which becomes important near the top of the barrier. However, it is clear from the quasiclassical analysis that this correction is approximate. In the quasiclassical approximation 


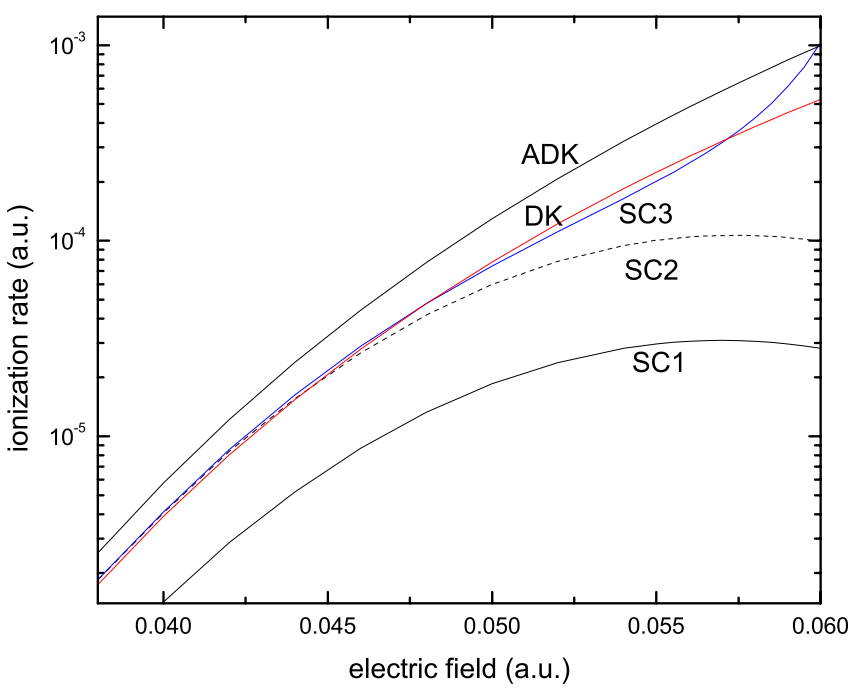

FIG. 3. (Color online) Ionization rate as a function of electric field for the hydrogen atom. Curve SC1-semiclassical calculation with unpolarized orbital and without inclusion of multiple reflections; curve SC2 - semiclassical with polarized orbital, but without multiple reflections; curve SC3 - semiclassical with multiple reflections; curve DK-exact quantal calculations [37]; curve ADKasymptotic (ADK) theory $[1,2]$.

the exponential expression of the type $\exp (-S)$ are valid only if $S$ is substantially higher than $\hbar$ (or 1 in a.u.). Near the top of the barrier the exact quantum-mechanical solution [35] in terms of the parabolic cylinder function should be used. In addition, the factor (41) contains the action corresponding to the shortest trajectory $(\rho=0)$ and therefore might overestimate the multiple reflection effect.

\section{RESULTS AND DISCUSSION}

\section{A. Ionization of the hydrogen atom}

To illustrate the above approach, we calculate the rate of ionization of the hydrogen atom. The formula of Smirnov and Chibisov in this case is reduced to the expression given by Landau and Lifshitz [36] (in a.u.)

$$
\Gamma=\frac{4}{F} \exp \left(-\frac{2}{3 F}\right) \text {. }
$$

Damburg and Kolosov [37] derived a more accurate expression which is very close to the exact numerical results at $F$ $<0.06$ a.u. (Note that the ionization becomes classically possible at $F=0.0625$ a.u.)

$$
\Gamma=4 R e^{-2 R / 3}\left(1-\frac{53}{12} F-\frac{3221}{288} F^{2}+O\left(F^{3}\right)\right)
$$

where

$$
R=(-2 E)^{3 / 2} / F,
$$

and the energy $E$ is calculated taking account of terms through $F^{2}$.

In Fig. 3 we present comparison of the semiclassical calculations with the asymptotic theory and exact quantum- mechanical results. We present three versions of the semiclassical calculations: the first uses an unperturbed groundstate hydrogen function, the second uses a polarized orbital, Eq. (36), but does not take into account multiple reflections at the classical turning points. The third takes into account the multiple reflection effect. It is apparent that the influence of the polarized orbital is very significant: polarization increases the initial probability density in the field direction and makes the decay rate substantially higher. The multiple reflections become significant at $F>0.048$ a.u. On the other hand, the approximate treatment of the multiple reflection effect leads to essential disagreement with exact quantal result at $F>0.057$ a.u.

It seems surprising that the asymptotic theory, which assumes an unperturbed orbital at the boundary of the atom, gives much higher value for the rate. As was discussed in the Introduction, one would expect that the ionization rate should be significantly suppressed in the asymptotic theory. Nevertheless, it, in fact, exceeds the exact result. Apparently the asymptotic theory strongly overestimates the preexponential factor in the expression for the decay rate.

\section{B. Ionization of complex atoms and molecules}

Turning to many-electron atoms and molecules, our goal will be to compare reliability of the asymptotic theory and to analyze a simple case of molecular suppression. Most experiments were performed with ac fields from laser pulses of finite duration, therefore comparison with experiments requires some additional calculations discussed, for example, in Refs. $[10,11]$. We will not present direct comparisons with experiments, but just assume that the relevant Keldysh parameter is small enough for the tunneling theory to be valid.

We continue to use the described one-electron approach except that polarized orbitals are calculated now numerically using conventional Gaussian basis Hartree-Fock calculations of molecules in an imposed electric field. The calculations involving $\mathrm{H}$ used a conventional $6-311+G$ basis, changing the standard scales to maximize the polarization energy. $\mathrm{Ar}$ calculations used a 6-311G $G^{* *}$ basis, also modified to maximize the polarization energy. The $6-311 G$ basis comprises six primitive Gaussians to represent the core orbital, and three functions for the valence orbitals each of which consists of 3,1 , and 1 primitive Gaussians. The $6-311+G$ basis is supplemented by diffuse functions of $s$ and $p$ type, and the 6-311 $G^{* *}$ basis by five $d$-type functions [38]. As an example, we present in Table I the exponential scale factors and orbital coefficients used for $\mathrm{H}_{2}$.

The GAMESS [39] and CRUNCH [40] program suites were used producing the necessary electric fields with two charges placed at large distances in the geometry of the calculation. The values of these charges and their positions relative to the molecule were changed to produce the field directions and field values needed.

If molecules are assumed to be randomly oriented, we average our result over molecular orientations relative to the field axis. In the case of a rare-gas atom we assume that only electrons from the outer $n p$ subshell are ionized, and calculate ionization rate for $\sigma$ and $\pi$ orbitals corresponding to the 
TABLE I. Gaussian basis for $\mathrm{H}_{2}$ calculation.

\begin{tabular}{lcc}
\hline \hline Designation & Exponential scale factor & Orbital coefficient \\
\hline $1 s$ & 68.1600 & 0.00255 \\
& 10.2465 & 0.01938 \\
& 2.34648 & 0.09280 \\
& 0.673320 & 0.29430 \\
$1 s^{\prime}$ & 0.224660 & 1.0 \\
$1 s^{\prime \prime}$ & 0.082217 & 1.0 \\
$1 s^{\prime \prime \prime}$ & 0.05 & 1.0 \\
$1 p$ & 1.0 & 1.0 \\
$1 p^{\prime}$ & 0.128 & 1.0 \\
\hline \hline
\end{tabular}

magnetic quantum numbers $m=0$ and $|m|=1$, respectively.

We will start first with the simplest molecule, $\mathrm{H}_{2}{ }^{+}$. Our basis generates a parallel polarizability of $\mathrm{H}_{2}^{+} \alpha_{\|}$ $=4.978$ a.u. and the perpendicular polarizability $\alpha_{\perp}=1.641$, which compares well with accurate $a b$ initio calculations of Bishop and Cheung [41], $\alpha_{\|}=5.078$ a.u., $\alpha_{\perp}=1.758$ a.u. We should add, however, that more recent calculations $[42,43]$ emphasize the importance of the nuclear motion for polarizability calculations. In particular, the polarizability of $\mathrm{H}_{2}^{+}$in the ground vibrational and rotational state is 3.168 a.u. compared to the polarizability of $\mathrm{D}_{2}^{+} 3.071$ a.u. and the fixednuclei value of Bishop and Cheung, 2.865 a.u. Note that in the fixed-nuclei limit the average polarizability $\bar{\alpha}$ is

$$
\bar{\alpha}=\frac{1}{3}\left(\alpha_{\|}+2 \alpha_{\perp}\right)
$$

Therefore our value is $\bar{\alpha}=2.754$ a.u. For our purposes, though, only the fixed-nuclei polarizability is required, since typically the tunneling time is much shorter than the rotational, or even vibrational, period, and the fixed-nuclei approximation appears to be quite accurate in this problem [23].

In Fig. 4 we present the ionization rate for $\mathrm{H}_{2}^{+}$in the ground electronic state. Both the case of randomly oriented molecules and molecules aligned along the electric field are shown. Since the $\sigma_{g}$ orbital in $\mathrm{H}_{2}{ }^{+}$is essentially isotropic, the difference is mainly due to the anisotropic feature of the polarizability. At weak fields our result for randomly oriented molecules is very close to the asymptotic theory whereas for higher fields our results are noticeably lower. This confirms the comparison of ADK with quantum complex rotation results presented by Tong et al. [10]. Still, in view of Fig. 1, the level of agreement between the asymptotic theory and accurate results with the correct tunneling probability is surprisingly good.

Turning to the $\mathrm{H}_{2}$ molecule, we obtain $\alpha_{\|}=6.481$ a.u. and $\alpha_{\perp}=4.321$ a.u. which can be compared to accurate calculations of Gough et al. [44] $\alpha_{\|}=6.762$ a.u. and $\alpha_{\perp}$ $=4.506$ a.u. This level of accuracy is about the same as that for $\mathrm{H}_{2}^{+}$.

The $\mathrm{H}_{2}$ case is perhaps the simplest example of molecular suppression. In this case the ionization rate for $\mathrm{H}_{2}$ is usually compared with that for atomic Ar. In Fig. 5 we present both

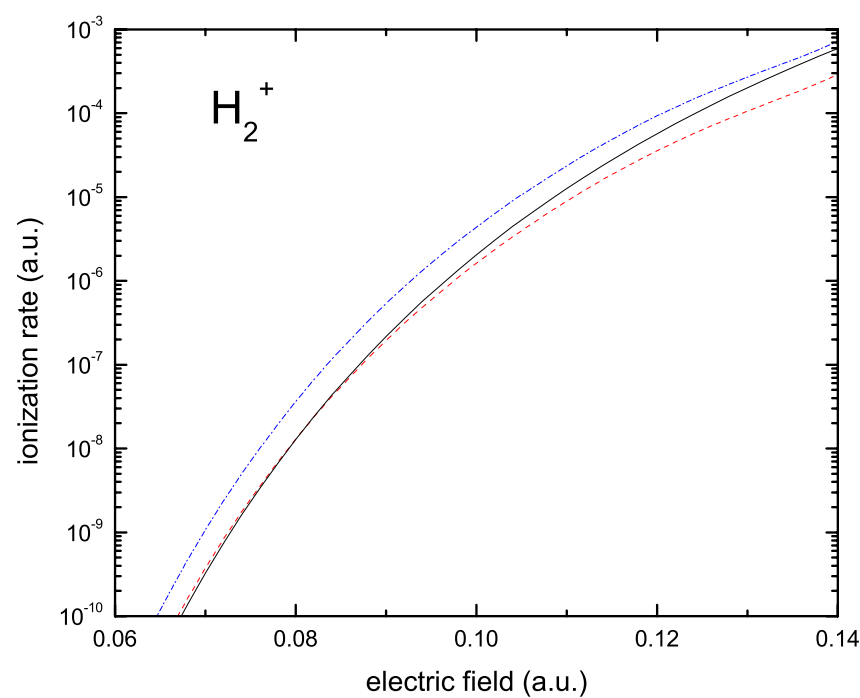

FIG. 4. (Color online) Ionization rate as a function of electric field for $\mathrm{H}_{2}^{+}$. Dashed-dotted curve-semiclassical calculation for the molecule aligned along the electric field; dashed curvesemiclassical calculation for random orientations; solid curveasymptotic (ADK) theory.

ionization rates and compare them with the results of the asymptotic theory. For $\mathrm{H}_{2}$ we used the vertical ionization potential, $|E|=16.35 \mathrm{eV}$, as suggested in Ref. [10]. Again, the asymptotic theory is surprisingly close to our calculations. Moreover, the suppression of ionization of the $\pi$ orbital in Ar, given in the asymptotic theory by the factor $F / \kappa^{3}$ (in a.u.) is about the same in our calculations. The reason for the molecular suppression is basically the same as that given by the asymptotic calculations [10]: the $3 p_{z}$ orbital in Ar has higher probability density in the field direction, increasing the tunneling probability, as compared to $\mathrm{H}_{2}$.

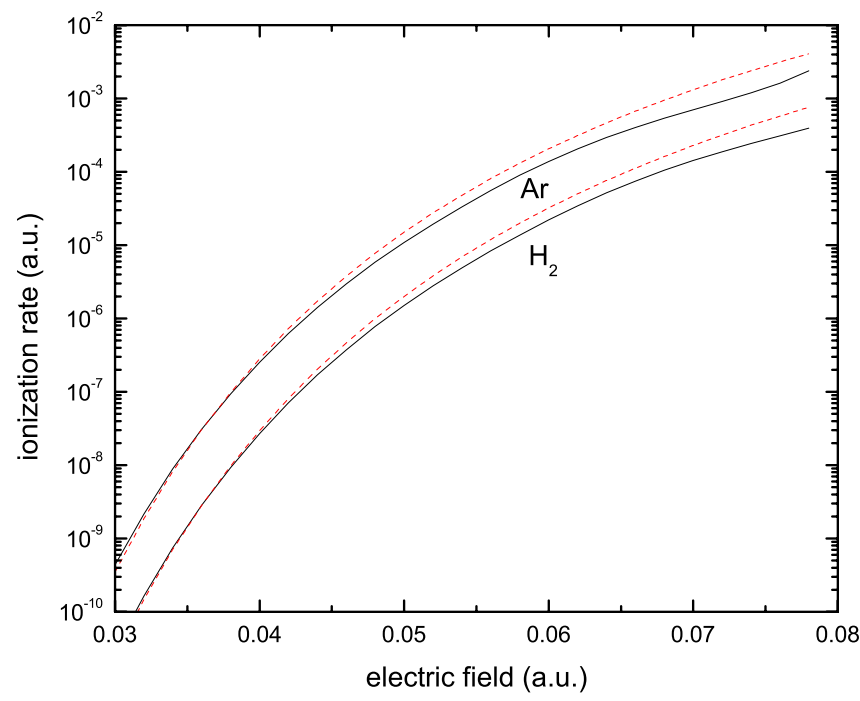

FIG. 5. (Color online) Ionization rate as a function of electric field for the Ar atom and $\mathrm{H}_{2}$ molecule. Solid curves-resent calculations; dashed curves-asymptotic (ADK) theory. 


\section{CONCLUSION}

We conclude that the asymptotic (ADK) theory gives unexpectedly good results for static-field ionization of atomic and simple molecular system. For the systems discussed, atomic hydrogen, $\mathrm{H}_{2}{ }^{+}, \mathrm{H}_{2}$, and $\mathrm{Ar}$ it is accurate within the factor of 2 even close to the barrier suppression region. Moreover, in the simplest case of molecular suppression, ionization of $\mathrm{H}_{2}$ versus Ar, it gives practically the same result as the more accurate semiclassical theory developed in the present paper. However, for more complicated molecular systems the situation is not that simple. In particular, the asymptotic theory is not able to explain experimental data on the $\mathrm{F}_{2}$ versus Ar case and some others. In the future we plan to investigate these systems by the method developed in the present paper.

Although the present treatment is limited by the fields below the barrier suppression region, it can be easily extended into the region above $F_{B S I}$ where the classical ionization is allowed. The range of fields in the vicinity of $F_{B S I}$ requires a quantum-mechanical approach. Our calculations suggest that this region starts at about 0.003 a.u. below $F_{B S I}$. Immediately above $F_{B S I}$ quantum effects associated with above-barrier reflection should be important.

\section{ACKNOWLEDGMENTS}

This work was supported by the National Science Foundation under Grant No. PHY-0652866.
[1] B. M. Smirnov and M. I. Chibisov, Zh. Eksp. Teor. Fiz. 49, 841 (1965); [Sov. Phys. JETP 22, 585 (1966)].

[2] A. M. Perelomov, V. S. Popov, and M. V. Terent'ev, Zh. Eksp. Teor. Fiz. 50, 1393 (1966); [Sov. Phys. JETP 23, 924 (1966)].

[3] M. V. Ammosov, N. B. Delone, and V. P. Krainov, Zh. Eksp. Teor. Fiz. 91, 2008 (1986); [Sov. Phys. JETP 64, 1191 (1986)].

[4] A. M. Perelomov and V. S. Popov, Zh. Eksp. Teor. Fiz. 52, 514 (1967); [Sov. Phys. JETP 25, 336 (1966)].

[5] T. D. G. Walsh, J. E. Decker, and S. L. Chin, J. Phys. B 26, L85 (1993).

[6] C. Guo, M. Li, J. P. Nibarger, and G. N. Gibson, Phys. Rev. A 58, R4271 (1998).

[7] A. Scrinzi, M. Geissler, and T. Brabec, Phys. Rev. Lett. 83, 706 (1999)

[8] C. Guo, Phys. Rev. Lett. 85, 2276 (2000).

[9] E. Wells, M. J. DeWitt, and R. R. Jones, Phys. Rev. A 66, 013409 (2002).

[10] X. M. Tong, Z. X. Zhao, and C. D. Lin, Phys. Rev. A 66, 033402 (2002).

[11] Z. X. Zhao, X. M. Tong, and C. D. Lin, Phys. Rev. A 67, 043404 (2003).

[12] B. Fabre, J. H. Posthumus, V. Adrianarijaona, J. Jureta, and X. Urbain, Laser Phys. 13, 964 (2003); X. Urbain, B. Fabre, V. M. Adrianarijaona, J. Jureta, J. H. Posthumus, A. Saenz, E. Baldit, and C. Cornaggia, Phys. Rev. Lett. 92, 163004 (2004).

[13] T. K. Kjeldsen and L. B. Madsen, Phys. Rev. A 71, 023411 (2005).

[14] V. P. Krainov and B. Shokri, Zh. Eksp. Teor. Fiz. 107, 1180 (1995); [JETP 80, 657 (1995)]; V. P. Krainov, J. Opt. Soc. Am. B 14, 425 (1997).

[15] A. Saenz, Phys. Rev. A 66, 063408 (2002).

[16] F. A. Ilkov, J. E. Decker, and S. L. Chin, J. Phys. B 25, 4005 (1992).

[17] L. V. Keldysh, Zh. Eksp. Teor. Fiz. 47, 1945 (1964); [Sov. Phys. JETP 20, 1307 (1965)].

[18] M. J. DeWitt and R. J. Levis, J. Chem. Phys. 108, 7045 (1998); 108, 7739 (1998).

[19] M. J. DeWitt and R. J. Levis, Phys. Rev. Lett. 81, 5101 (1998).

[20] T. D. G. Walsh, F. A. Ilkov, J. E. Decker, and S. L. Chin, J.
Phys. B 27, 3767 (1994).

[21] A. Talebpour, S. Larochelle, and S. L. Chin, J. Phys. B 31, L49 (1998).

[22] A. Talebpour, C.-Y. Chien, and S. L. Chin, J. Phys. B 29, L677 (1996).

[23] A. Saenz, J. Phys. B 33, 4365 (2000).

[24] J. Muth-Böhm, A. Becker, and F. H. M. Faisal, Phys. Rev. Lett. 85, 2280 (2000).

[25] M. C. Gutzwiller, J. Math. Phys. 8, 1979 (1967).

[26] W. H. Miller, J. Chem. Phys. 53, 1949 (1970); R. A. Marcus, ibid. 54, 3965 (1971).

[27] J. B. Delos, Adv. Chem. Phys. 65, 161 (1986); M. L. Du and J. B. Delos, Phys. Rev. A 38, 1896 (1988).

[28] A. M. Perelomov, V. S. Popov, and A. M. Terent'ev, Zh. Eksp. Teor. Fiz. 51, 309 (1966) [Sov. Phys. JETP 24, 207 (1966)]; V. S. Popov, V. P. Kuznetsov, and A. M. Perelomov, ibid. 53, 331 (1967); [Sov. Phys. JETP 26, 222 (1967)]; V. S. Popov, ibid. 61, 1334 (1971); [Sov. Phys. JETP 34, 709 (1971)]; V. S. Popov, B. M. Karnakov, and V. D. Mur, Phys. Lett. A 229, 306 (1997).

[29] V. S. Popov, Yad. Fiz. 68, 717 (2005); [Phys. At. Nucl. 68, $686(2005)]$.

[30] W. H. Miller and T. F. George, J. Chem. Phys. 56, 5668 (1972).

[31] V. P. Maslov and M. V. Fedoriuk, Semiclassical approximation in quantum mechanics (Reidel, Boston, 1981).

[32] I. I. Fabrikant, Zh. Eksp. Teor. Fiz. 79, 2070 (1980) [Sov. Phys. JETP 52, 1045 (1980)].

[33] C. Lanczos, Z. Phys. 62, 518 (1930); G. F. Drukarev, Zh. Eksp. Teor. Fiz. 75, 473 (1978) [Sov. Phys. JETP 48, 237 (1978)].

[34] A. Temkin, Phys. Rev. 116, 358 (1959); A. Temkin and J. C. Lamkin, ibid. 121, 788 (1961).

[35] V. V. Kolosov, J. Phys. B 16, 25 (1983).

[36] L. D. Landau and E. M. Lifshitz, Quantum Mechanics (Nonrelativistic theory) (Pergamon, Oxford, 1979).

[37] R. J. Damburg and V. V. Kolosov, J. Phys. B 11, 1921 (1978).

[38] F. Jensen, Introduction to Computational Chemistry (Wiley, Chichester, 1999).

[39] M. W. Schmidt, K. K. Baldridge, J. A. Boatz, S. T. Elbert, M. S. Gordon, J. H. Jensen, S. Koseki, N. Matsunaga, K. A. 
Nguyen, S. J. Su, T. L. Windus, M. Dupuis, and J. A. Montgomery, J. Comput. Chem. 14, 1347 (1993).

[40] G. A. Gallup, R. L. Vance, J. R. Collins, and J. M. Norbeck, Adv. Quantum Chem. 16, 229 (1982).

[41] D. M. Bishop and L. M. Cheung, J. Phys. B 11, 3133 (1978).
[42] J. Shertzer and C. H. Green, Phys. Rev. A 58, 1082 (1998).

[43] J. M. Taylor, A. Dalgarno, and J. F. Babb, Phys. Rev. A 60, R2630 (1999).

[44] K. M. Gough, M. M. Yacowar, R. H. Cleve, and J. R. Dwyer, Can. J. Chem. 74, 1139 (1996). 